\title{
Experimental Confirmation of Quantum Monodromy: The Millimeter Wave Spectrum of Cyanogen Isothiocyanate NCNCS
}

\author{
Brenda P. Winnewisser, Manfred Winnewisser, Ivan R. Medvedev, Markus Behnke, and Frank C. De Lucia \\ Department of Physics, The Ohio State University, Columbus, Ohio 43210-1106, USA \\ Stephen C. Ross \\ Department of Physics and Centre for Laser, Atomic, and Molecular Sciences, University of New Brunswick, \\ P.O. Box 4400, Fredericton, New Brunswick E3B 5A3, Canada \\ Jacek Koput \\ Department of Chemistry, Adam Mickiewicz University, 60-780 Poznan, Poland
}

(Received 22 August 2005; published 5 December 2005)

\begin{abstract}
We have made energy-momentum maps for the experimental end-over-end rotational energy and the two-dimensional bending vibrational energy, both of which confirm the dominating effects of nontrivial quantum monodromy in cyanogen isothiocyanate. Accidental resonances in the rotational spectra yield accurate intervals between bending states.
\end{abstract}

DOI: $10.1103 /$ PhysRevLett.95.243002

The concept of monodromy (Greek for "once around") in a champagne bottle potential was introduced in 1991 by Bates [1]. For a two-dimensional anharmonic oscillator with a potential function resembling the bottom of a champagne bottle, the hump represents a "gross topological obstruction." The top of the barrier is called the critical or monodromy point. Child and collaborators [2-4] have translated the abstract mathematics into the language of quantum mechanics and spectroscopy. They pointed out via classical trajectory calculations that the sense of precession for a particle changes sign abruptly if the trajectory moves from below the monodromy point to above the monodromy point.

In search of a molecule with an isotropic twodimensional anharmonic potential function with more than one bending state below its monodromy point we rediscovered cyanogen isothiocyanate, NCNCS, the microwave spectrum of which was measured and partially assigned in a masterful analysis by King, Kroto, and Landsberg in 1985 [5]. The central bending potential function was shown from the initial analysis of the microwave data to have a barrier height to linearity of about $270 \mathrm{~cm}^{-1}$ [6] with a low-lying bending mode around $90 \mathrm{~cm}^{-1}$. This implies that we should be able to probe rovibrational energy states both below and above the monodromy point.

The $r_{e}$ structure was calculated according to the coupled-cluster single double (triple) $[\mathrm{CCSD}(\mathrm{T})]$ and correlation-consistent polarized valence quintuple zeta $[\mathrm{cc}-\mathrm{pV}(5+d) \mathrm{Z}]$ level of theory with corrections for the core-correlation effects at the $\operatorname{CCSD}(T) / c c-$ $\mathrm{pCV}(\mathrm{Q}+\mathrm{d}) \mathrm{Z}$ level. The structure, displayed in Fig. 1, shows a planar chainform molecule. The components of the permanent electric dipole moment at this configuration are calculated at the $\operatorname{CCSD}(\mathrm{T}) / \mathrm{cc}-\mathrm{pV}(\mathrm{Q}+\mathrm{d}) \mathrm{Z}$ level to be $\mu_{\mathrm{a}}=3.20 \mathrm{D}$ and $\mu_{\mathrm{b}}=0.41 \mathrm{D}$.
PACS numbers: 31.15.Ar, 31.50.Bc, 33.15.Hp, 33.20.Bx

The fundamental vibrational wave numbers for NCNCS, which has molecular symmetry $C_{s}$, are given in Table I. The harmonic wave numbers were calculated at the $\operatorname{CCSD}(\mathrm{T}) / \mathrm{cc}-\mathrm{pV}(\mathrm{T}+\mathrm{d}) \mathrm{Z}$ level of theory. Anharmonic corrections calculated at the MP2/cc $-\mathrm{pV}(\mathrm{T}+\mathrm{d}) \mathrm{Z}$ level of theory were added to yield the anharmonic wave numbers. The large amplitude bending motion, as can be seen from Fig. 1 and Table I, takes place about the central N atom.

Following the method of King and Kroto [7] the sample of NCNCS was prepared by the pyrolytic isomerization reaction of $\mathrm{S}(\mathrm{CN})_{2}$ vapor in a flow system directly attached to the absorption cell at a pressure of 5 mTorr.

The spectroscopic measurements were carried out in the frequency range from 115 to $365 \mathrm{GHz}$ using the fast scan submillimeter spectroscopic technique incorporating recent improvements [8,9]. The accuracy of the absorption line positions is estimated the be $\pm 50 \mathrm{kHz}$.

The present data set contains rotational transitions arising from NCNCS molecules in the ground vibrational state and 5 excited bending states of the $\nu_{7}$ mode, which we label $\nu_{b}$ indicating the use of bent $(b)$ notation while $\nu_{l}$ will refer to linear $(l)$ notation. As we shall see, both notations

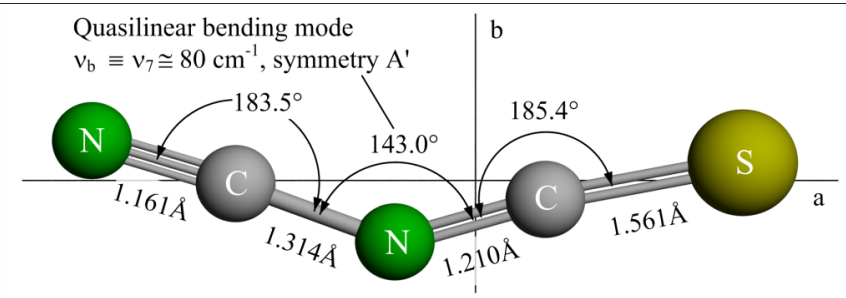

FIG. 1 (color online). Ab initio predicted equilibrium structure of NCNCS. 
TABLE I. A $b$ initio predicted harmonic and anharmonic fundamental vibrational modes for NCNCS (Symmetry $\mathrm{C}_{s}$ ).

\begin{tabular}{|c|c|c|c|c|}
\hline & $\Gamma$ & $\omega^{\mathrm{a}}$ & $\nu^{\mathrm{a}}$ & $\begin{array}{l}\text { Harmonic potential energy distribution } \\
\qquad[\mathrm{N}(1) \mathrm{C}(2) \mathrm{N}(3) \mathrm{C}(4) \mathrm{S}(5)]\end{array}$ \\
\hline$\nu_{1}$ & $A^{\prime}$ & 2302 & 2258 & $73 \% 1-2 s^{\mathrm{b}}, 22 \% 2-3 s$ \\
\hline$\nu_{2}$ & $A^{\prime}$ & 2058 & 2024 & $74 \% 3-4 s, 15 \% 4-5 s, 10 \% 1-2 s$ \\
\hline$\nu_{3}$ & $A^{\prime}$ & 1188 & 1178 & $47 \% 2-3 s, 41 \% 4-5 s, 13 \% 1-2 s$ \\
\hline$\nu_{4}$ & $A^{\prime}$ & 688 & 662 & $39 \% 4-5 s, 31 \% 2-3 s, 25 \% 3-4 s$ \\
\hline$\nu_{5}$ & $A^{\prime}$ & 483 & 472 & $68 \% 3-4-5 b, 19 \% 1-2-3 b, 11 \% 2-3-4 b$ \\
\hline$\nu_{8}$ & $A^{\prime \prime}$ & 452 & 454 & $65 \% 2-3-4-5 t, 35 \% 1-2-3-4 t$ \\
\hline$\nu_{6}$ & $A^{\prime}$ & 444 & 447 & $74 \% 1-2-3 b, 25 \% 3-4-5 b$ \\
\hline$\nu_{9}$ & $A^{\prime \prime}$ & 430 & 429 & $65 \% 1-2-3-4 t, 35 \% 2-3-4-5 t$ \\
\hline$\nu_{7}$ & $A^{\prime}$ & 89 & 80 & $94 \%$ 2-3-4 large amplitude bend \\
\hline
\end{tabular}

${ }^{\mathrm{a}} \omega$ stands for harmonic wave number in $\mathrm{cm}^{-1}, \nu$ for anharmonic wave number in $\mathrm{cm}^{-1}$.

${ }^{\mathrm{b}} s$ stands for stretching, $b$ for bending, $t$ for torsional vibration.

are applicable to the vibrational eigenstates of NCNCS; the vibrational quantum number $v_{l}$ is related to $v_{b}$ via the relation $v_{l}=2 v_{b}+\left|K_{a}\right|$. The asymmetric rotor angular momentum quantum number $K_{a}$, the projection of the total angular momentum $\mathbf{J}$ on the axis of least moment of inertia, $a$, is identical with $|\ell|$ in the linear molecular model.

All rotational transitions discussed here were assigned to their respective $J$ values using the Loomis-Wood option of the CAAARS assignment package [9] and adjusting the coefficients in a conventional power series in $J(J+1)$ for each value of $v_{b}$ and $K_{a}$. The effective rotational constant $B_{\text {eff }}$ multiplies the leading term $J(J+1)$ in each polynomial, representing the dominant contribution to the end-over-end rotation $\left[B_{\text {eff }} \approx(B+C) / 2\right.$ for a bent molecule, $B$ for a linear molecule]. Assignments of $K_{a}$ were obtained by combining the information from the microwave study [5] and predictions from the general semi-rigid bender (GSRB) Hamiltonian calculations [6]. Furthermore, line intensities and asymmetry (or $\ell$-type doubling) splittings for $K_{a}=1,2$, and 3 were essential.

The key features of the GSRB $[6,10]$ Hamiltonian are a kinetic energy representation of rotation and the large amplitude bend, and a semi-rigid contribution, allowing for the dependence of internuclear distances and end bond angles on the large amplitude bending coordinate. Only atomic masses are fixed; good starting values for the internuclear distances and end angles and for the bending potential function parameters are required. The structural and potential parameters and semirigidity parameters are adjusted in a fit to pure rotational or rovibrational data. For a detailed discussion the reader is referred to Refs. [6,11].

The map of end-over-end rotational energy, represented by $B_{\text {eff }}$, versus the angular momentum quantum number $\pm K_{a}\left(K_{a}\right.$ is used here as in the classical sense), given in Fig. 2, displays the consequences of quantum monodromy in NCNCS. The experimental $\Delta B_{\text {eff }}$ values are listed in Table II. (The values for asymmetry doublets are aver- aged.) From the dependence of the $B_{\text {eff }}$ values on $v_{b}$ and $K_{a}$, it is obvious that neither the Hamiltonian for an asymmetric rotor molecule nor the Hamiltonian for a linear molecule is applicable over the entire range.

The available data for NCNCS includes no experimental $E\left(K_{a}, v_{b}\right)$ values. However, the GSRB Hamiltonian [11] allowed us to calculate the vibrational energy levels associated with the quasilinear bending mode $\nu_{b}$ given in Table III and thus to plot the graph for NCNCS shown in Fig. 3. This plot has precisely the topology predicted in the work of Bates [1] and Child [3] for a system with monodromy: The smooth, parabolic (blue online) curves in Fig. 3 representing the states $v_{b}=0,1,2$, evolve rapidly to a succession of curves with sharp kinks at $K_{a}=0$ beginning with the monodromy point at $v_{b}=3$.

A quantitative confirmation of the accuracy of the GSRB predictions is provided by several local resonances observed in the pure rotational spectrum. For example, as indicated in Fig. 3, a $\Delta v_{b}=+1, \Delta K_{a}=-3$ local Coriolis resonance interaction has been observed around $J=80$ between the bending states $v_{b}=2, K_{a}=4^{e}$ and $v_{b}=3$, $K_{a}=1^{e}$. The superscript ${ }^{e}$ designates the parity. Though separated by about $5 \mathrm{~cm}^{-1}$ at low $J$, the two series of levels

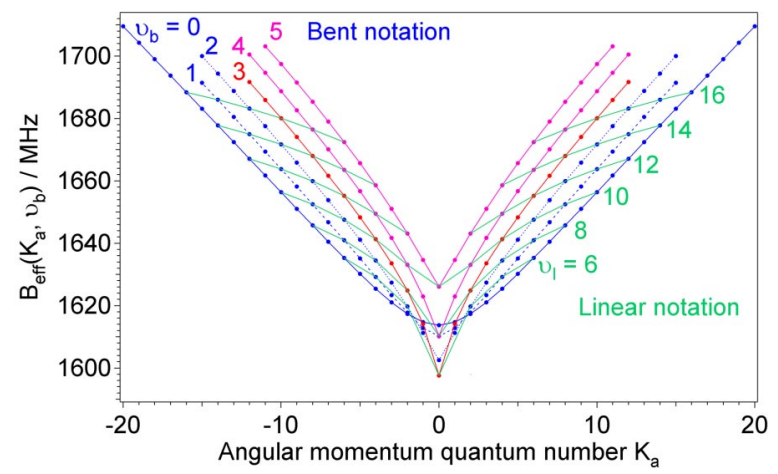

FIG. 2 (color online). Experimentally determined $B_{\text {eff }}$ values for NCNCS plotted versus $K_{a}$. 
TABLE II. Experimentally determined differences in rotational constants $\Delta B_{\text {eff }}$ in $\mathrm{MHz}$ for NCNCS. $\Delta B_{\text {eff }}=\left[B_{\text {eff }}\left(K_{a}, v_{b}\right)-B_{\text {eff }}(0,0)\right]$ with $B_{\text {eff }}(0,0)=1613.7186 \mathrm{MHz}$.

\begin{tabular}{rrrrrrr}
\hline \hline$K_{a}$ & $v_{b}=0$ & \multicolumn{1}{c}{$\boldsymbol{v}_{b}=1$} & \multicolumn{1}{c}{$\boldsymbol{v}_{b}=2$} & \multicolumn{1}{c}{$\boldsymbol{v}_{b}=3$} & \multicolumn{1}{c}{$\boldsymbol{v}_{b}=4$} & $\boldsymbol{v}_{b}=5$ \\
\hline 0 & 0.0000 & -3.5075 & -11.1772 & -16.1337 & -3.4351 & 12.3032 \\
1 & 0.9807 & -0.8821 & -2.4494 & 0.3744 & 9.2121 & 20.8636 \\
2 & 3.6019 & 4.0253 & 6.0088 & 11.1143 & 19.3301 & 29.3654 \\
3 & 7.3238 & 9.6602 & 13.6557 & 19.8371 & 27.9588 & 37.2838 \\
4 & 11.7077 & 15.4394 & 20.7407 & 27.5632 & 35.7309 & 44.8478 \\
5 & 16.4984 & 21.3595 & 27.4372 & 34.5905 & 42.9530 & 51.9393 \\
6 & 21.5298 & 27.2128 & 33.8673 & 41.4517 & 49.7988 & 58.7228 \\
7 & 26.7094 & 32.9975 & 40.0970 & 47.9303 & 56.3726 & 65.2702 \\
8 & 31.9818 & 38.7398 & 46.1747 & 54.2069 & 62.7267 & 71.6465 \\
9 & 37.2949 & 44.4310 & 52.1315 & 60.3218 & 68.9231 & 77.8242 \\
10 & 42.6339 & 50.0758 & 57.9895 & 66.3108 & 74.9724 & 83.7249 \\
11 & 47.9872 & 55.6757 & 63.7636 & 72.1985 & 80.8818 & 89.3891 \\
12 & 53.3404 & 61.2335 & 69.4709 & 77.9984 & 86.7637 & \\
13 & 58.6910 & 66.7533 & 75.1110 & & & \\
14 & 64.0313 & 72.2374 & 80.7062 & & & \\
15 & 69.3616 & 77.6932 & 86.2514 & & & \\
16 & 74.6778 & & & & & \\
17 & 79.9810 & & & & & \\
18 & 85.2724 & 90.5513 & & & &
\end{tabular}

come to coincide at $J=80$ because of differing $B_{\text {eff }}$ values. A preliminary analysis shows that the values in Table III are accurate to better than $1 \mathrm{~cm}^{-1}$. The evaluation of more of these resonances will allow a further refinement of the GSRB calculation.

Using the information describing the potential function derived from the GSRB for the quasilinear bending mode of NCNCS we obtain Fig. 4. This figure shows that the $K_{a}=0$ level of the $v_{b}=3$ state is just barely above the classical monodromy point.

Figure 2 reveals that NCNCS molecules in the $K_{a}=0$ level of the $v_{b}=3$ state feature the smallest effective rotational constant and therefore the largest moment of

TABLE III. Calculated term values $E\left(K_{a}, v_{b}\right) / h c$ in $\mathrm{cm}^{-1}$ for the large amplitude bending mode $\nu_{b}$ of NCNCS using the GSRB Hamiltonian. $E(0,0) / h c=42.6 \mathrm{~cm}^{-1}$.

\begin{tabular}{rrrrrrrr}
\hline \hline$K_{a}$ & $v_{b}=0$ & $v_{b}=1$ & $v_{b}=2$ & $v_{b}=3$ & $v_{b}=4$ & $v_{b}=5$ & $v_{b}=6$ \\
\hline 0 & 0.0 & 84.6 & 162.2 & 231.4 & 304.0 & 389.1 & 484.2 \\
1 & 3.6 & 89.7 & 172.4 & 254.1 & 340.1 & 432.8 & 532.8 \\
2 & 13.9 & 103.6 & 192.5 & 282.7 & 376.9 & 476.3 & 581.1 \\
3 & 30.2 & 124.2 & 218.8 & 315.6 & 416.1 & 520.7 & 629.8 \\
4 & 51.9 & 150.2 & 249.9 & 352.1 & 457.6 & 566.8 & 679.6 \\
5 & 78.3 & 180.7 & 284.9 & 391.7 & 501.5 & 614.5 & 730.7 \\
6 & 110.0 & 215.2 & 323.4 & 434.1 & 547.6 & 664.0 & 783.2 \\
7 & 143.3 & 253.0 & 364.9 & 479.1 & 595.8 & 715.2 & 837.1 \\
8 & 181.0 & 294.0 & 409.1 & 526.4 & 646.1 & 768.2 & 892.4 \\
9 & 221.8 & 337.7 & 455.7 & 575.9 & 698.2 & 822.7 & 949.1 \\
\hline \hline
\end{tabular}

inertia. This effect was observed for the first time during the analysis of $\mathrm{CH}_{3} \mathrm{NCS}[12,13]$ a quasisymmetric top, which actually has two states below the monodromy point, and again in the quasilinear molecules $\mathrm{BrCNO}$ and $\mathrm{ClCNO}$ [11], both of which have one $K_{a}=0$ state well below the monodromy point. In the case of all of these molecules, the pronounced drop in $B$-value for low $K_{a}$ values near the top of the barrier is the result of the quantum mechanical or classical time average of the moment of inertia. This effect is most clearly seen to be systematic in the case of NCNCS.

The topology of the bending potential function is indeed distinctively mapped onto the observed spectrum and thus

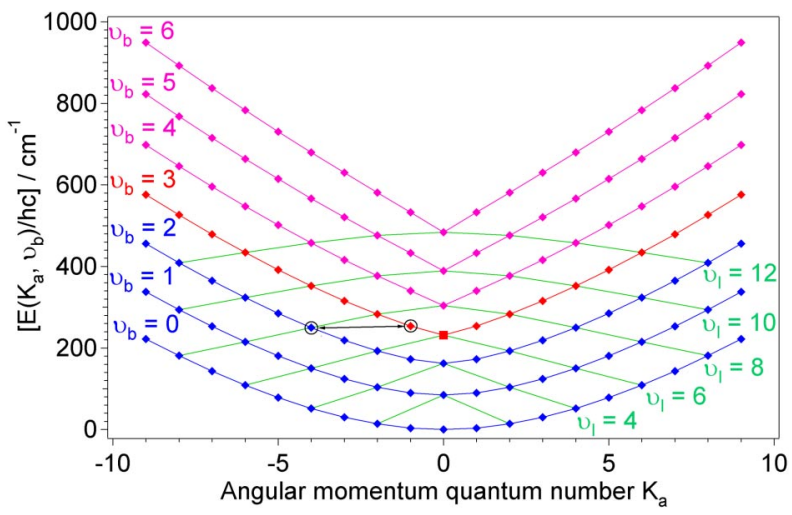

FIG. 3 (color online). Quantum monodromy plot of calculated $v_{b}$ bending vibrational levels. The color scheme follows Fig. 2 . A local Coriolis resonance is indicated between the two bending states $v_{b}=2, K_{a}=4^{e}$ and $v_{b}=3, K_{a}=1^{e}$. 


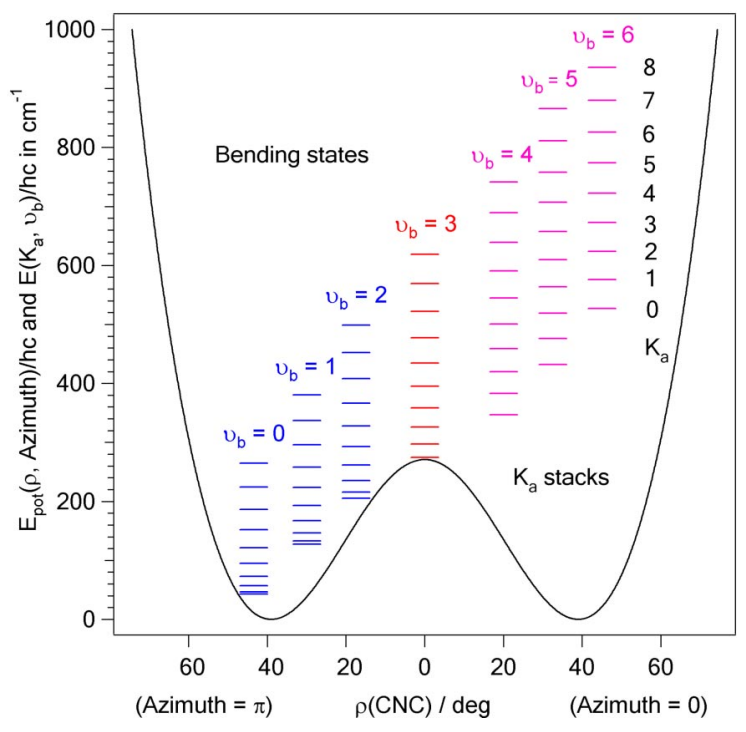

FIG. 4 (color online). Cut through the effective twodimensional bending potential energy function $E_{\text {pot }}(\rho$, Azimuth $) / h c$ of the mode $\nu_{b} \equiv \nu_{7}$ for NCNCS. The bending coordinate $\rho(\mathrm{CNC})$ is defined as the supplement of the CNC bond angle. The vibrational term values $E\left(K_{a}, v_{b}\right) /$ $h c$ are also entered.

onto any representation of the rovibrational energy versus $K_{a}$. A representation of the end-over-end rotational energy, as in Fig. 2, accentuates the anomalies observed in the basic monodromy plot of $E\left(K_{a}, v_{b}\right)$ versus $K_{a}$ for NCNCS given in Fig. 3. Since the results we present here were first reported [14], Tennyson and coworkers [15] have managed to assign very highly excited levels in $\mathrm{H}_{2} \mathrm{O}$ which exhibit energy-momentum characteristics similar to those shown here, also confirming the pattern imposed by quantum monodromy.

The first recognition that interesting minima can be seen by plotting rovibrational term values of a three-atomic molecule against $K_{a}$, as it passes from a bent to linear configuration, must be attributed to Dixon's $\mathrm{NH}_{2}$ work [16]. The topological perspective has revealed further systematic properties of such systems. With our extension of this approach to the $B_{\text {eff }}$ values, we believe that we have introduced, in addition, an aid for the assignment of the complex rotational structure in spectra of quasilinear molecules.

We thank J. Tennyson and M. Child for stimulating discussions. The experimental work at OSU is supported by the Army Research Office, and the GSRB work by the Natural Sciences and Engineering Research Council of Canada.

[1] L. M. Bates, J. App. Math. Phys. 42, 837 (1991).

[2] M. S. Child, T. Weston, and J. Tennyson, Mol. Phys. 96, 371 (1999).

[3] M. S. Child, J. Phys. A 31, 657 (1998).

[4] M. S. Child, J. Mol. Spectrosc. 210, 157 (2001).

[5] M. A. King, H. W. Kroto, and B. M. Landsberg, J. Mol. Spectrosc. 113, 1 (1985).

[6] S. C. Ross, J. Mol. Spectrosc. 132, 48 (1988).

[7] M.A. King and H.W. Kroto, J. Chem. Soc. Chem. Commun. 1980, 606 (1980).

[8] I. Medvedev, M. Winnewisser, F. C. DeLucia, E. Herbst, E. Białkowska-Jaworska, L. Pszczółkolwski, and Z. Kisiel, J. Mol. Spectrosc. 228, 314 (2004).

[9] I. R. Medvedev, M. Winnewisser, B.P. Winnewisser, F. C. DeLucia, and E. Herbst, J. Mol. Struct. 742, 229 (2005).

[10] S. C. Ross, T. A. Cooper, S. Firth, H. W. Kroto, and D. R. Walton, J. Mol. Spectrosc. 152, 152 (1992).

[11] H. Lichau, C. W. Gillies, J.Z. Gillies, S.C. Ross, B. P. Winnewisser, and M. Winnewisser, J. Phys. Chem. A 105, 10065 (2001).

[12] J. Koput, J. Mol. Spectrosc. 118, 189 (1986).

[13] J. Koput, J. Mol. Spectrosc. 118, 448 (1986).

[14] B. P. Winnewisser, M. Winnewisser, I. R. Medvedev, M. Behnke, F. C. DeLucia, S. Ross, and J. Koput, in 60th International Symposium on Molecular Spectroscopy, Columbus, $\mathrm{OH}, 2005$, Report No. TH07.

[15] N. F. Zobov, S. V. Shirin, O. L. Polyansky, J. Tennyson, P. F. Coheur, P.F. Bernath, M. Carleer, and R. Colin, Chem. Phys. Lett. (to be published).

[16] R. N. Dixon, Trans. Faraday Soc. 60, 1363 (1964). 